\title{
Active Contour-Based Multiresolution Transforms for the Segmentation of Fluorescence Microscope Images
}

\author{
Gowri Srinivasa $^{1}$, Matthew Fickus ${ }^{3}$ and Jelena Kovačević ${ }^{1,2}$ \\ ${ }^{1}$ Center for Bioimage Informatics and Dept. of Biomedical Engineering \\ ${ }^{2}$ Dept. of Electrical and Computer Engineering, \\ Carnegie Mellon University, Pittsburgh, PA, USA \\ ${ }^{3}$ Dept. of Mathematics and Statistics, \\ Air Force Institute of Technology, Wright-Patterson AFB, OH, USA
}

\begin{abstract}
In recent years, the focus in biological science has shifted to understanding complex systems at the cellular and molecular levels, a task greatly facilitated by fluorescence microscopy. Segmentation, a fundamental yet difficult problem, is often the first processing step following acquisition. We have previously demonstrated that a stochastic active contour based algorithm together with the concept of topology preservation (TPSTACS) successfully segments single cells from multicell images. In this paper we demonstrate that TPSTACS successfully segments images from other imaging modalities such as DIC microscopy, MRI and fMRI. While this method is a viable alternative to hand segmentation, it is not yet ready to be used for high-throughput applications due to its large run time. Thus, we highlight some of the benefits of combining TPSTACS with the multiresolution approach for the segmentation of fluorescence microscope images. Here we propose a multiscale active contour (MSAC) transformation framework for developing a family of modular algorithms for the segmentation of fluorescence microscope images in particular, and biomedical images in general. While this framework retains the flexibility and the high quality of the segmentation provided by active contour-based algorithms, it offers a boost in the efficiency as well as a framework to compute new features that further enhance the segmentation.
\end{abstract}

\section{INTRODUCTION}

Fluorescence microscopy is one of the main techniques used by biologists to observe processes in a living cell, greatly facilitated by the advent of new fluorescent probes such as the green fluorescent protein. These probes are introduced into the cell and then the cell is imaged using a florescence microscope. The fluorescence microscope can be used to collect 2D slices and 3D volumes (2D slices along the z-axis) as well as image the cell at multiple time points and using different parallel channels leading to a large number of multidimensional data sets.

\subsection{Biological Motivation: Automating Knowledge Extraction in Fluorescence Microscopy}

Visual processing of such a large number of multidimensional images is inefficient, not reproducible, timeconsuming and error-prone, prompting the movement towards automated, efficient and robust processing of such biological images. Moreover, some information hidden in the images may not be easily discerned by the human eye. Thus, we strive towards automated processing, not only for speed and efficiency, but to generate new knowledge through use of sophisticated algorithms as well. While such tools are widely present in clinical (medical) imaging, their use is not as widespread in imaging of biological systems at cellular and molecular levels.

A Must: Automated Segmentation. Segmentation is often the first step after acquisition. It is a fundamental, yet very difficult, problem in image processing, especially for microscope images which contain more than one cell. The goal of segmentation is to separate objects of interest from other objects, as well as from the background and is used, for example, in high-content screening to identify cellular structures. While it is desirable for all tasks dealing with biological or biomedical images to be as automated as possible, this requirement is absolutely crucial for segmentation. For example, it takes a skilled expert one hour to segment an image as in Fig. 1 and almost three days for an image as in Fig. 5. Possibly the only automated algorithm currently used in fluorescence microscopy is watershed segmentation (see Section 1.2.1), while state-of-the-art algorithms from image processing have only begun to be applied (see Section 1.2.2). 
In this section we introduce two biological problems that highlight the need for automated segmentation. This is because hand segmentation of a sufficient number of images might not be feasible and does not ensure the reproducibility and accuracy of results. While our focus is on segmentation of fluorescence microscope images, we consider a wider range of modalities, including problems using differential interference contrast microscopy (DIC), magnetic resonance imaging (MRI) and functional MRI (fMRI), to show the broader impact automated segmentation would have.

\subsubsection{Determining Protein Subcellular Location}

Proteins are integral components of cells that impact their functioning. One of the crucial aspects of a protein's activity and function is its subcellular location. Today's method of choice to determine protein subcellular location is fluorescence microscopy. Murphy pioneered automated interpretation and analysis of protein subcellular location images, resulting in systems that can classify protein location patterns with well-characterized reliability and better sensitivity than human observers. ${ }^{1,2}$

In one of the data sets used in Ref. 3, the cells had been marked for a specific protein along with probes to obtain parallel images of the total protein and total DNA. This data was segmented using the seeded watershed algorithm on the total-protein channel, using the nuclei as seeds. As we will see in Section 1.2.1, the seeded watershed algorithm suffers from both splits (one cell/object is split into more than one) as well as merges (more than one cell/object are merged into a single one) forcing a large fraction (nearly $62 \%$, see Section 2.1.1) of the cells to be discarded thus wasting time and resources. ${ }^{4}$

\subsubsection{The Influence of Golgi-Protein Expression on the Size of the Golgi Apparatus}

The Golgi apparatus is an organelle that mediates the processing and sorting of proteins and lipids in the final stages of their biosynthesis. The cytoplasmic surface of the Golgi apparatus is also a site for numerous important signaling pathways. Defects in membrane trafficking are responsible for many human diseases. Since an understanding of the molecular basis of these defects is paving the way to new therapeutics, a study of the Golgi apparatus is of particular interest.

The research of Linstedt et. al. focuses on the aspects that influence and control the size of the Golgi apparatus. ${ }^{5}$ These tests depend on a quantitative assay comparing Golgi size to cell size. The accurate segmentation of the images obtained from a fluorescence microscope assay is the critical step in determining cell volume. In the absence of an automated segmentation algorithm that works satisfactorily, the tests involve hand segmenting the cell boundaries in each of as many as 20 optical slices. For this reason, the analysis has been limited to fewer optical slices of the cells and to small numbers of cells. ${ }^{6}$ To extend their experimental findings, the analysis of many more cells and much larger numbers of slices per cell is necessary. The automated segmentation algorithm developed here will allow a rigorous, objective and efficient means of Golgi/cell volume determination.

\subsubsection{Importance of Automated Segmentation on Other Modalities}

While we focus on developing the tools needed for segmentation of fluorescence microscope images, due to their flexibility, we believe these tools will allow the development of algorithms for automated segmentation on other modalities as well. As representative examples, we discuss some of the projects for which our collaborators are currently looking for segmentation solutions.

DIC Images of Yeast. The yeast organism has become one of the most often used eukaryotic microorganisms for biological studies. One of the standard ways of imaging yeast is using differential interference contrast (DIC) microscopy. It provides an excellent way for showing contrast in transparent specimens. One of the most important uses of DIC microscopy is that it represents an optical view of the entire cell and some of its parts, whereas any fluorescence label will exhibit a certain localization pattern (which may be restricted and could also vary over time). DIC microscopy does not require UV illumination (which may damage cells) and it works well on both live and fixed samples. Furthermore, it requires no special reagents (antibodies, cDNAs, dyes, etc.). Segmentation is the first step in quantitative and automated studies of yeast. Due to the typically large number of these cells in each image (see Fig. 3), hand segmentation is highly impractical and time consuming. Only recently has an attempt been made to automate the segmentation of this data. ${ }^{7}$ 
MRI Images of the Heart. MRI has emerged as a powerful technique to noninvasively visualize biological structures. Cardiac MRI is being used by Laine and his team to understand heart motion and quantify the leftventricular heart volume with the motivation of being able to realize more accurate ejection fraction measures. ${ }^{8}$ The segmentation of this type of images (see Fig. 4) can also drive techniques to detect abnormal wall motion due to ischemia. A particular time-series of this data set has been successfully segmented by adapting the STACS algorithm to this data (see Section 2.1.3). A challenge in this project is to extend the technique to work on the peripheral volumes that have poorer signal quality and are beset with noise.

fMRI Images of the Brain. With the development of functional MRI techniques, it is now possible to directly visualize neuronal activity in the brain by using the amount of oxygen in the surrounding blood flow as a proxy for neuronal activity. This, in turn, can be used to study the development, structure and activity of the human brain. In particular, the work of Wandell and his team focuses on using functional neuro-imaging to study visual pathways. ${ }^{9}$ To generate meaningful visualizations of the data obtained from fMRI studies, however, it is important to first obtain accurate topological maps of the area of the brain in which the neuronal activity is being measured - the gray matter in the cerebral cortex. One way to create these topological maps is to segment 3D MRI images of the brain into three regions: gray matter, white matter and cerebral spinal fluid. Once the $3 \mathrm{D}$ topology of the gray matter region has been identified by segmentation, an equivalent $2 \mathrm{D}$ visualization can be created by flattening out the 3D shape. The flattened 2D representation makes it easier to visualize the deep furrows (sulcii) that exist in the gray matter.

\subsection{Segmentation Algorithms}

Problem Statement. As highlighted in the previous subsection, segmentation is a fundamental task in image processing. Since we discuss the segmentation of real-valued discrete images of size $M \times N$ (similarly for higher dimensional data sets), segmentation may be defined as the mapping $\Psi: f \mapsto \Omega$, that is, a partitioning of the pixels in image $f \in \mathbb{R}^{M \times N}$ to a mask $\Omega=\bigcup \Omega_{i}$, with $\bigcap \Omega_{i}=\emptyset$. These masks, $\Omega_{i}$, are indicator functions of an object of interest such that any pixel identified as an object in $f$ belongs to one and only one $\Omega_{i}$. The mask $\Omega^{\mathrm{c}}$ represents the background. $\Omega$ may be represented as a black and white mask of the same size as the input image, such that the white regions correspond to the objects of interest and the black to the background. Another representation of $\Omega_{i}$ may be a multi-hued mask with a different color assigned to each object (member of a distinct $\Omega_{i}$ ) and black to the background.

In case of segmentation of biological images, $\Psi$ is a good mapping if the resulting $\Omega_{i}$ are biologically meaningful segments of the input image. The standard of comparison to quantify the quality of the mapping is normally the output of manual segmentation.

\subsubsection{Current Segmentation Method in Fluorescence Microscopy: Seeded Watershed}

One of the most widely used algorithms for the segmentation of fluorescence microscope images is a region-based segmentation algorithm known as the Seeded Watershed $(\mathrm{SW}) .{ }^{10}$ It works by interpreting the image as elevation in a landscape splitting the image into regions, similar to the drainage regions of this landscape. To create the watersheds, a gradient magnitude image is built, in which water will start to rise from minima representing areas of low gradient (such as areas inside the cell or in the background regions), and the watershed borders will be built at the maxima of the gradient magnitude. Thus, ideally, the borders will be at the edges of cells, assuming those edges are well defined. However, as this is not the case for fluorescence microscope images, the contour (watershed border) keeps evolving following gradual changes in the gradient, resulting in segmented regions much larger than the true cell. Various modifications of the watershed algorithm have been extensively used to segment single cells from multicell images. ${ }^{11}$

Problems with Watershed Segmentation. The problems with this algorithm are twofold. Firstly, many segmented regions are discarded due to true cells being segmented into more than one region (splits), and more than one cell being merged into the same region (merges, see Fig. 1(c)). Any postprocessing to deal with merges would typically be manual. Secondly, by design, the seeded watershed algorithm splits the image into regions and does not define a contour around the region of interest. The segmented regions are typically much larger than the true cell (same figure), leading to a fair amount of background noise outside the cell being included with the cell. 


\subsubsection{Active Contours: State-of-the-Art in Segmentation}

Active contour algorithms are the state-of-the-art in segmentation. In particular, the Stochastic Active Contour Scheme belongs to the class of geometric active contour algorithms and it was originally developed to segment heart MR images. ${ }^{12}$

Given an image $f(x, y)$, the level-set function $\phi(x, y, t)$ is positive inside the contour $C$, zero on $C$ and negative outside. ${ }^{13}$ To evolve the contour, we evolve the level-set function itself. To represent the templates of the image pixels that are inside, outside or on the contour, we define masks using the regularized Heavyside function $\mathcal{H}(\phi)$, with value one well outside the contour ( $\phi$ is positive and much larger than some small $\epsilon$ ) and zero inside the contour (where $\phi$ is negative and much larger then some small $\epsilon$ ). Let $\delta(\phi)=\mathrm{d} \mathcal{H}(\phi) / \mathrm{d} \phi$. We can now use the functions $\mathcal{H}(\phi), 1-\mathcal{H}(\phi)$ and $\delta(\phi)$ to extract the pixels inside, outside and on $C$, respectively. ${ }^{12,13}$

As a key concept, the segmentation problem is mapped into an energy functional minimization problem,

$$
\text { minimize: } J(C)=\sum_{i}^{N} \lambda_{i} J_{i}(C),
$$

where $\lambda_{i}$ is the weight of energy functional $J_{i}$. Thus, in this setting, we can have any number of energy functionals chosen appropriately based on the application. For example, in the STACS algorithm $N=4$. We have $J_{1}$ is the contour smoothing term, $J_{2}$ incorporates the prior knowledge on the shape of the contour, $J_{3}$ is called the region-based term and $J_{4}$ is an edge-based term, with corresponding weights, $\lambda_{I}, \lambda_{2}, \lambda_{3}$, and $\lambda_{4}$, respectively, that control the relative strength of the four terms. The minimum of the functional is found at a zero of its first variation $\delta J(C)=0$, from which a partial differential is extracted of the form $F(C)=0$, termed the Euler-Lagrange equation. It is usually solved by introducing an artificial time parameter $t$ into $C(x, y, t)$, and solving

$$
\frac{\partial C}{\partial t}=F(C) .
$$

In steady state, $\partial C / \partial t=0$, leading to the solution of the Euler-Lagrange equation by introducing an explicit parameter, $t$, as

$$
\frac{\partial}{\partial t} \phi(x, y, t)=\sum_{i} \lambda_{i} F_{i}(x, y, t)
$$

For STACS, a specific form of the equation is obtained with $F_{1}$ as the internal force, $F_{2}$ the external force, $F_{3}$ a curvature force and $F_{4}$ a shape-based force, with weights, $\lambda_{1}, \ldots, \lambda_{4}$, respectively. Active contours have been used extensively in medical imaging and are only recently finding their way into biological imaging. ${ }^{14,15}$

\section{ACTIVE CONTOUR-BASED ALGORITHMS}

In this section we review the algorithmic results that demonstrate our success in segmenting fluorescence microscope cell images as well as its flexibility in incorporating new forces for segmenting data from some other modalities.

\subsection{The bimagicLab STACS Segmentation Algorithms for Fluorescence Microscopy \\ 2.1.1 TPSTACS: Algorithm for Segmentation of Protein Subcellular Location Images}

Herein we present only the pertinent details of the algorithm we developed for segmentation of fluorescence microscope images introduced in Section 1.1.1. Further details can be obtained from Ref. 4,16.

Data Set. Two channels (total DNA and total protein) in HeLa cells expressing GFP-UCE were imaged using confocal immunofluorescence microscopy. ${ }^{3}$ Serial sections in the z-axis through entire cells were taken with a step size of $0.1628 \mu \mathrm{m}$ and a pixel size of $0.0977 \mu \mathrm{m}$ in the $\mathrm{x}$ and $\mathrm{y}$ dimensions $(1024 \times 1024$ pixels per section). The total number of images was 82 for $83 \mathrm{D}$ z-stacks. We were also provided the masks resulting from the seeded watershed (SW) algorithm.

Modified STACS. While the original STACS uses four forces: region-based, edge, shape prior and contour smoothness, given in (3), in our modified version, we use only two: an external, region-based force $F_{r}$ and an 
internal force $F_{c}$ which depends on the curvature of the contour. We describe the evolution of the level set function as

$$
\frac{\partial \phi(\mathbf{x}, t)}{\partial t}=\lambda_{r} F_{r}(\mathbf{x})+\lambda_{c} F_{c}(\mathbf{x}, t),
$$

where $\lambda_{r}$ and $\lambda_{c}$ are scalar coefficients that weigh the region-based force and the curvature force, respectively.

The external or region-based force is based on the assumption that the pixels in the objects and the background are drawn from two different statistical models: $M_{1}$ and $M_{2}$. Thus, a pixel lying inside the contour $C$ should be described by the model $M_{1}$, while a pixel outside the contour should be described by $M_{2} \cdot{ }^{12}$ More specifically, our external region-based force relies on the idea that the density of proteins should be higher inside the cell than outside. The internal force is a smoothing force based on the curvature $F_{c}$ of the contour. At each iteration the magnitude of this force is computed from the level-set function as described in Ref. 13.

In (4), the coefficients $\lambda_{r}$ and $\lambda_{c}$ evolve in time: this is called an annealing, or, cooling schedule. ${ }^{12}$ (These names come from a parallel problem in statistical mechanics and is different from the annealing commonly referred to in optimization problems.) Experiments reveal that the region-based coefficient $\lambda_{r}$ should decrease with time, whereas the curvature coefficient $\lambda_{c}$ should remain constant.

One of the important steps in the algorithm is the initialization of the contour. Equation (4) assumes that an initial level set function is available. To obtain it, we perform an edge-based segmentation on the DNA channel to develop initial contours. Then we initialize the level set function using the Euclidean distance transform. Given a binary mask as input, for each pixel in the plane, we assign the shortest Euclidean distance between the pixel and the nearest point on a contour as its value. Finally, we invert the sign of the distance for pixels outside of the contour.

Evolution of the level set to obtain the final contour is done by introducing an artificial time parameter and numerically solving the Euler-Lagrange equation as described in (3), with the appropriate forces for this data. As the forces are computed only at the points on the contour, they must be extended to the entire domain in order to suitably update the level set function. This is done for every pixel in the image by computing the closest point to it on the contour and simply replicating the force at that point. ${ }^{13}$ The extent of evolution is controlled by a parameter that specifies the number of iterations a priori.

We compare our results to those obtained by SW. We also have hand-segmented (HS) total-protein images which we use as ground truth. As the edges in these images are poorly defined, there are many instances of large areas of noise and hand segmentation is often imprecise and varies from person to person. Thus, although we are comparing our results to HS contours, it is possible that the algorithm actually performs correctly while disagreeing with the HS contours.

Results. The results of this algorithm are depicted in Fig. 1(d). The algorithm automatically produced continuous smooth contours that appear to match well with the HS contours Fig. 1(b). However, we do encounter problems; when cells are close together or linked by an area of noise, merges occur. This happens because geometric active contours handle change in topology, that is, contours merging and splitting, gracefully. Since

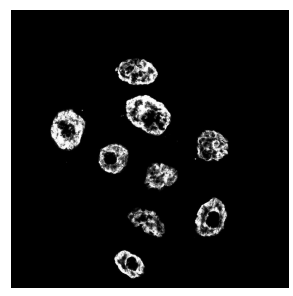

(a)

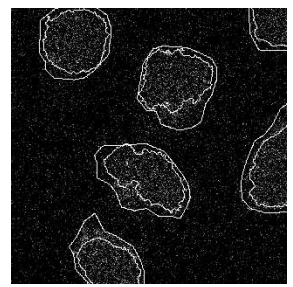

(b)

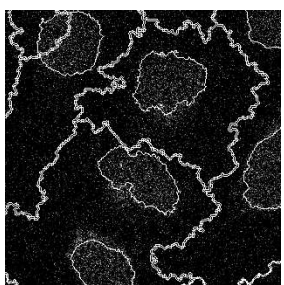

(c)

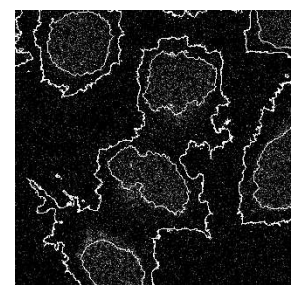

(d)

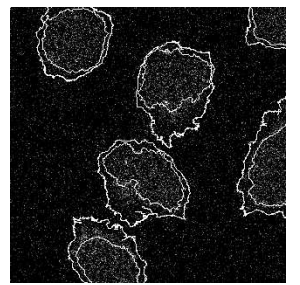

(e)

Figure 1. Fluorescence microscope images used to determine protein subcellular location: (a) The DNA channel and the cropped regions of the segmented total-protein image using (b) hand segmentation, (c) seeded watershed, ${ }^{3}$ (d) modified STACS and (e) TPSTACS. ${ }^{4,16}$ 
we know the number of cells (given by the number of nuclei in the DNA channel), we want to impose a constant number of contours on our algorithm (termed "topology preservation").

Topology Preserving STACS. We conserve the topology or the number of contours obtained after initialization (which correspond to the number of cells detected in the image) over the course of the evolution of the level-set function by detecting and not changing the sign of the nonsimple points. ${ }^{17}$

Measures of Performance. To assess the performance of our algorithm, we use three common methods: area overlap, area similarity and recall/precision. We compare SW and TPSTACS to the hand-segmented images (HS), as well as DNA images.

Area Overlap (AO) detects how much of each cell overlaps with HS (or DNA). This measure will count the splits although the resulting contour might be discarded. This measure is lenient towards algorithms producing loose contours, such as SW.

Area Similarity $(A S)^{18}$ compares the area of that mask with the area of any SW/TPSTACS mask which overlaps with it and normalizes it by the total area of both masks (unlike area overlap). Thus, this measure penalizes an algorithm if its contour is not tight, even though it might contain the entire hand-segmented contour.

The Recall/Precision $(R / P)$ method derives two quantities as percentages. They depend on a true positive, $T^{(+)}$— a mask designated by the algorithm as well as the HS mask, a false positive, $F^{(+)}$—a mask designated as a cell but which is not and, similarly, a false negative, $F^{(-)} . R$ tells us how many of the contours were correctly drawn and $P$ tells us how many of the contours drawn were correct.

\begin{tabular}{llcc}
\hline & & SW [\%] & TPSTACS [\%] \\
\hline Area Similarity (AS) & & 30.82 & 80.51 \\
\hline Area Overlap (AO) & HS & 62.15 & 82.14 \\
& DNA & 62.29 & 99.80 \\
\hline Recall (R) & HS (T=70\%) & 37.88 & 71.13 \\
Precision (P) & DNA (T=95\%) & 36.75 & 99.06 \\
& HS (T=70\%) & 39.99 & 76.82 \\
& DNA (T=95\%) & 36.28 & 99.06 \\
\hline
\end{tabular}

Table 1. Segmentation results for the seeded watershed algorithm (SW) and TPSTACS. The algorithms were tested against both hand-segmented images (HS) as well as the DNA ones (DNA). Note that $R$ and $P$ are given for the value of threshold $T=70 \%$ for HS and $T=95 \%$ for DNA (from Ref. 4 ).

Results. We ran our algorithm on the above set of images and set the cooling parameters to experimentally determined values. $\lambda_{c}$ was constant and equal to one and $\lambda_{r}$ decreased from 50 to 10 with the iteration. This means that the region-based term dominates in the beginning, growing the contours to roughly divide the pixels based on their statistical models, while the curvature term remains constant and grows in relative importance towards the end, smoothing the contour. To assess the performance of our algorithm, we use $A O, A S$ and $R / P$. We compare SW and TPSTACS to the hand-segmented images HS, as well as the DNA images. Results for both the seeded watershed and TPSTACS are given in Table 1 as well as Fig. 1(e). All measures have been averaged over all cells and all images. ${ }^{4}$

The AS measure yielded $80.51 \%$ for TPSTACS versus $30.82 \%$ for SW; this was expected as TPSTACS produces tight contours as opposed to SW ones (see Fig. 1(e) and (c), respectively). Area overlap yielded $82.14 \%$ for TPSTACS versus $62.15 \%$ for SW (against HS). Looking at the area overlap against the DNA (as well as recall and precision against the DNA), we see that essentially, almost $100 \%$ of our segmented cells are usable. Therefore, by both objective measures of quality (recall/precision, area similarity and area overlap) as well as subjective (visual inspection), we conclude that TPSTACS outperforms SW by a fair margin.

Discussion. Despite the impressive results, there are a few limitations to the applicability of TPSTACS. For instance, in its present design it cannot work without an initial contour. Thus, in cases where there is no DNA channel or any equivalent that affords an easy detection of the approximate location and number of cells, the algorithm cannot segment the total-protein image. 
Further, the algorithm is extremely time consuming with the complexity growing nonlinearly with the size of the image. A profile of the implementation reveals that nearly $70 \%$ of the computation load comes from the function that extends the values of the force (computed at the points on the contour) to the entire domain in order to update the level-set function at each iteration. Optimization approaches to speed up the evolution of the level set function include the narrow-band approach. ${ }^{17}$ Since our objects of interest - the cells - are not regular in shape, it was computationally infeasible to create the narrow-band around the evolving contour.

LATPSTACS. We developed a local area version of the algorithm to restrict the region of level-set update to a small rectangular neighborhood around the cell that retains an average $A S=98 \%$ with the TPSTACS results. ${ }^{16}$ The savings in run time were not significant. Furthermore, when the density of cells is high, the local area implementation very quickly degenerates to the original problem.

Towards Multiresolution. The issues of initialization and computational complexity point, at first blush, to a method that is able to ideally work on a smaller version of the image (to reduce the computations of the extension function). Such a requirement is consistent with the well-established and widely applied theory of multiresolution. ${ }^{19}$ MR transformations are known to offer localization, adaptivity and speed. For example, our team showed that using MR for classification of fluorescence microscope data significantly improves the classification accuracy, demonstrating the presence of hidden information in multiresolution subspaces. ${ }^{2}$

One might imagine we could quickly perform some initial segmentation on a very coarse version of our image and refine on the finer versions. Ideas along these lines have already appeared..$^{20-23}$ The advantage of speed is obtained from the fact that multiresolution transformations are computationally economical and the reduction in the size of the image itself reduces the computational load imposed by the extension function described above. The details of this method as applied to the total protein (HeLa-UCE) images is presented in Ref. 16. As a proof of concept for the applicability of this method to cell images, we present the results on the data for which the TPSTACS algorithm could not be used. The following subsection briefly describes this effort.

\subsubsection{MRSTACS: Algorithm for Segmentation of Images to Study Golgi Proteins}

Data Set. The images for this problem (which was introduced in Section 1.1.2) were acquired by the Linstedt team and consist of 15 z-stacks of 40 images each of size $1024 \times 1344$. There is a Golgi-protein channel and a parallel channel in which the cells' membrane proteins are marked. In this data set, we do not have the DNA channel.

As there may be multiple Golgi bodies in a cell, unlike the DNA which appears as a single unit (when the cell is not undergoing mitotic division), the Golgi channel cannot be used in the same fashion as the DNA channel for initialization. Thus the same algorithm developed for the subcellular protein location problem cannot be used. Furthermore, while TPSTACS has been tested extensively on the total-protein channel, the images in this data set have been stained with a dye targeting membrane proteins, so the signal can be expected to be sparser than, or at least not as uniform as the protein subcellular location images.

MRSTACS. We used a 2D analysis filter bank with the simplest MR filters, the Haar basis, and performed a 3-level decomposition. We expect (a) the downsampling to greatly reduce the run time and (b) the smoothing to offer cells easier to discern. To initialize the contours, we apply a threshold to detect the cells in the transformed image, followed by morphological operators to refine that detection. With fair initial estimates, a few iterations of the algorithm are sufficient to get a good coarse segmentation in the transform domain. We then interpolate the contours and refine the results by iterating a few times at each higher level. We use topology preservation to prevent any merges in the contour evolution stage.

Results. We use the same forces and values of the parameters $\lambda_{r}$ and $\lambda_{c}$ as TPSTACS and recompute the model statistics only. Due to the subsampling and good initialization due to smoothing, both provided by MR, the number of iterations required is much less. With this method (see an example in Fig. 2), we compute an initial coarse segmentation in less than 10 seconds and the refined final contours in less than 30 minutes (on an Intel Pentium M 1.6 GHz platform) as opposed to nearly 4-6 hours using the traditional TPSTACS method.

Discussion. Note that we currently interpolate the contour on the coarse level by replicating the nearestneighbor values in the evolving level set. This might result in jagged contours. Using a smoother interpolation method such as bicubic interpolation runs the risk of merging contours at the interpolation stage despite the 


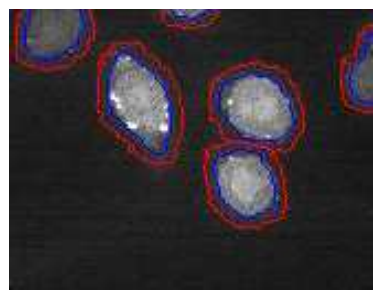

(a)

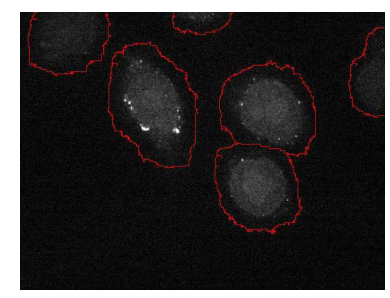

(b)

Figure 2. (a) Coarse segmentation at level three. The blue contour indicates the result of the blob detection and the red contour the coarse segmentation obtained after iterating TPSTACS on the coarsest subspace. (b) Segmentation on the original image.

topology preservation method that is applied at the curve evolution stage. Further venues of exploration are studying the preservation of topology across the scales and understanding the effect of different filters on different images and finding the optimal number of decomposition levels for a given data set.

\subsection{3 xxSTACS: Algorithms for Segmentation on Other Modalities}

We recognize that the segmentation demands posed by some of the images obtained from other imaging modalities overlap enormously with those that underlie the design of TPSTACS. While TPSTACS was designed primarily for the segmentation of fluorescence microscope images, as noted in Section 1.2.2, (1) allows us to include any number of forces adapted to the data. Given below are some of the applications of the algorithm, with suitable adaptations within the framework, to segment images from other modalities.

DIC Microscopy Images of Yeast. We now discuss segmentation of yeast images introduced in Section 1.1.3.

Data Set. We are given a set of closely spaced cells (yeast colonies) and are required to segment the individual cells in the DIC channel (Fig. 3(a)). The data set also has a parallel DNA channel and a GFP labelled specific protein channel. $^{24}$

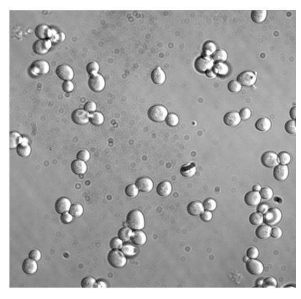

(a)

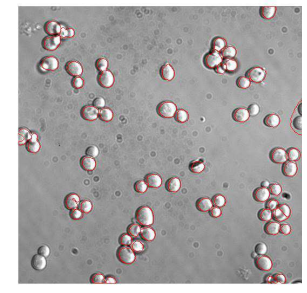

(b)

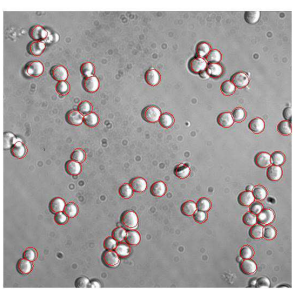

(c)

Figure 3. (a) A DIC microscope image of yeast cells, (b) segmentation results after automated initialization and (c) segmentation result after a manual initialization.

Algorithm. As the information in the parallel DNA information is ambiguous and insufficient to yield an initial contour we cannot use it to initialize the contour. Likewise the specific protein channel is of no avail to us for initialization. We initialize the contour automatically by first distinguishing the clumps of cells from the background by thresholding the gradient image. Next, we remove blobs with holes larger than a pre-determined size as invalid objects as well as very small fragments that are unlikely to be cells. This results in clumps of cells that are still mostly merged. Using a combination of k-means clustering and the watershed algorithm, we merge the fragmented portions of a cell, while splitting spuriously merged cells.

Evolution of the contour is based on the region-based force and the edge-based force (3). While the intensity difference between the foreground and background is usually weak, in the DIC channel, the edges are quite pronounced. Thus, the influence of the edge force is designed to increase with the iteration number. The edge maps can also be used as a stopping criterion to prevent the contours from finding the background. Further, using topology preservation maintains the number of contours detected in the initialization stage, preventing merges to which this data is highly prone. 
Results. Experimentation reveals that the challenge in this data is the initialization itself as we tend to get a good segmentation if we are able to first detect the presence of individual cells. Fig. 3(b), shows a representative result of the segmentation with automated initialization. Since the procedure is highly dependent on the initialization, Fig. 3(c) shows the result with a manual initialization.

Discussion. The automated initialization requires significant tuning before it can be robust and work accurately. With a purely automated version, we often end up mistakenly splitting cells due to the high variation in intensity, while failing to detect a few legitimate cells. Thus there is much scope for improvement in the initialization phase. The accurate performance of the algorithm after manual initialization suggests that the procedure would benefit immensely from a minimal user interaction (such as marking a single point in each cell).

MRI Images of the Heart. The task is to segment the heart walls - endocardium and epicardium-from a 2D time series of MR images of a human chest cavity, the problem we introduced in Section 1.1.3.

Data Set. We are given a set of $25 \mathrm{MRI}$ images of the heart about $40 \mathrm{~ms}$ apart and so correspond roughly to one heartbeat of a clinically healthy volunteer.

Algorithm. The endocardium usually appears brighter than most structures in the chest cavity. Thus, the initial contour for an image is obtained by thresholding the image. Mere thresholding, however, picks up some other structures. To get rid of these spurious detections, we use the fact that the endocardium is the most circular of these structures. During the course of evolving the contour, we use topology preservation as there is only one endocardium (as well as only one epicardium). In fact, the region-based force and curvature force used in TPSTACS, work successfully on this data. Since the initialization detects almost all of the endocardium, just a few iterations of the modified TPSTACS algorithm are sufficient to refine the contour and add the missing portions. The final contour obtained for the endocardium serves as the initial contour for the segmentation of the epicardium. The same algorithm with the same parameters (but with appropriately tuned statistical models for the region based force) is iterated a few times to segment the epicardium.

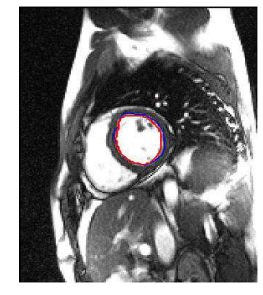

(a)

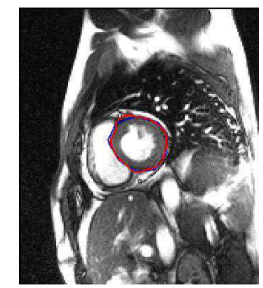

(b)

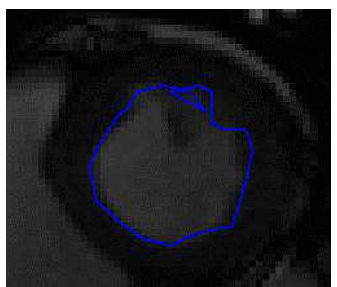

(c)

Figure 4. (a) Segmentation of the endocardium and (b) epicardium. (Red contour: TPSTACS. Blue contour: handsegmented ground truth.)(c) An example of a self-loop and nonsmooth contour in the hand-segmented ground truth.

Results. A representative image of the outcome of the endocardium segmentation is shown in Fig. 4(a). The outcome of the epicardium segmentation is shown in Fig. 4(b). We observe that the algorithm works well even when there is no edge information present. An entire frame of size $192 \times 156$ is completely segmented with a high degree of accuracy in less than fifteen seconds. The average area similarity compared with the hand-segmented ground truth is $93 \%$ for the endocardium and $94 \%$ for the epicardium.

Discussion. The higher accuracy for epicardium segmentation despite the relatively lower accuracy for its initial contour - the endocardium segmentation - could be explained as follows. For a few of the frames, the discrepancy between the contour from the algorithm and the ground truth is due to the inconsistency and/or inaccuracy in the hand segmented contour (see Fig. 4(c)) rather than any inaccuracy on the part of the algorithm. Though the ground truth is provided by an expert, such anomalies are expected as the process of hand segmenting the images is a tedious one.

Some of the future directions include tuning the algorithm to be robust to noise in the data in peripheral sectioning planes and also in case of data from high-speed MR imaging or in ultrasound images that are also used in such studies. Further, we can also use the time series information and cyclic nature of the heartbeat to our advantage in designing the algorithm. 
fMRI Images of the Brain. In brain fMRI images (see Section 1.1.3), the functional activity under study is detected in the gray matter, which is obtained by growing layers of it on top of segmented white matter.

Data Set. We report the results of testing our algorithm on the MRI images of a child brain made available to us by the VISTA laboratory at Stanford University. It consists of 400 images - 100 in the coronal plane, 140 in the sagittal and 160 in the axial sectioning planes - each of size $217 \times 180$ pixels. The VISTA lab also provided us with human expert-based segmentation for the left hemisphere to evaluate our algorithm.

Algorithm. The challenge in segmenting such images stems from the highly convoluted nature of the gray matter. While voxel-based classification methods that use image statistics and histogram-based thresholding are partially successful, they are affected by intensity variations and the need to maintain distinct templates for various brain pathologies. ${ }^{25}$ Active-contour-based methods, on the other hand, have considerable advantages due to their topological flexibility. ${ }^{26}$ An automated initial segmentation used by the Stanford team results in several anomalies which necessitate hours of involved manual postprocessing to clean up the results. ${ }^{27}$ The specific goal of this project is to improve the accuracy of segmentation so as to significantly reduce, if not eliminate entirely, the need for manual postprocessing, and thus reduce wasted resources both in terms of money and time.

We obtain an initial contour for the white matter segmentation by thresholding the image and the curve evolution is based solely on the region-based force. To take advantage of the available 3D information, we consider the 2D segmentation results for every voxel in three different planes - axial, coronal and sagittal and segment by majority voting.

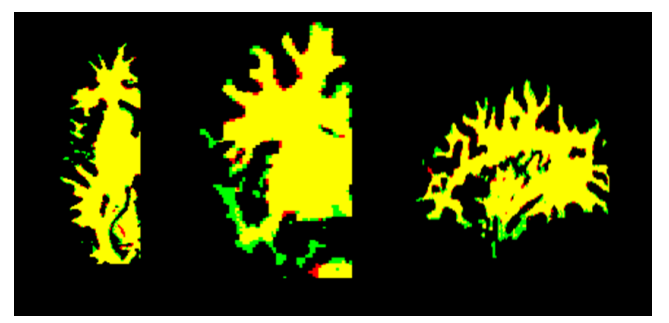

Figure 5. L to r: Results of segmenting the white matter of the left-hemisphere in the axial, coronal and sagittal planes. The yellow/red/green labels correspond to true positives/false positives/false negatives, respectively.

Results. A qualitative assessment of the segmentation outcome is made by visual inspection. Fig. 5 shows the segmentation along the three sectioning planes. Table 2 reports a quantitative assessment of four possible implementations: voting-based segmentation (Voting), segmentation based on axial slices only (Axial), segmentation based on coronal slices only (Coronal) and segmentation based on sagittal slices only (Sagittal). From the table we note that the voting-based technique achieves the best performance on each performance measure.

\begin{tabular}{lccc}
\hline & $T^{(+)}(\%)$ & $F^{(+)}(\%)$ & $A S(\%)$ \\
\hline Axial & 86.26 & 2.32 & 82.83 \\
Coronal & 89.02 & 2.55 & 80.76 \\
Sagittal & 86.21 & 1.79 & 81.92 \\
Voting & 89.13 & 1.63 & 85.07 \\
\hline
\end{tabular}

Table 2. Quantitative measures of the segmentation performance of Voting-Based STACS. $T^{(+)}$indicates true positives that is, pixels correctly identified as belonging to the white matter region, $F^{(+)}$indicates false positives that is, pixels incorrectly identified as white matter and $A S$ denotes area similarity.

Discussion. Typically, an $A S \geq 70 \%$ is considered excellent agreement with the ground truth. ${ }^{28}$ Thus we note that the outcome of the voting-based STACS algorithm for fMRI images is in good agreement with the ground truth provided to us. It is noteworthy, however, that the algorithm takes nearly 36 hours to segment nearly 600 images. While this is still faster and less tedious than three days of manual labor, which is what it takes in the absence of an automated method, the run time is still quite high. 


\subsection{Towards Multiscale Active Contour Transforms}

Examining what we have done so far, we can say that the active contour methods work on fluorescence microscope data (as well as on MRI, fMRI and DIC microscopy) fairly well and certainly better than the watershed algorithm. This success is primarily due to the flexibility they offer (choosing any combination of available forces) as well as on the new, stochastic ideas. ${ }^{12}$ The stochastic (region-based) term in (3) is the only term that specifically responds to the stochastic nature of fluorescence microscope data sets. The success we have had in applying one of the simplest multiresolution ideas in this framework also demonstrates that bringing state-of-the-art methods from image processing into fluorescence microscopy is a worthwhile and long overdue effort.

As the domain of the level-set function is the whole image, the algorithm is extremely slow. Even MR as applied to this framework in Section 2.1.2 is not a solution to the expensive computation that is costly by design. Though there have been some attempts in the recent past to introduce active contour algorithms to the segmentation of biological images they are designed for the specific task on hand. Further, akin to the constraints used in our own extension function for TPSTACS, there are a lot of rules that are defined to ensure the system works. While they do perform satisfactorily, the rules - such as a particle once burnt cannot be resurrected to avoid self intersections - are rather ad-hoc. There is no systematic way of coming up with such constraints for every new data set encountered. Moreover, it is not even an elegant way of developing a concept that obviously holds immense potential.

These considerations motivate us to revisit the mathematical set up underlying the design. We attempt to get rid of the extension function (essentially a version of the all-pairs-shortest-path problem) which does not have any obvious or truly beneficial optimization. Next, the huge advantage of the geometric embedding of the active contour algorithm is its nonparametrization. In the present scenario, this benefit is all but lost as by explicitly working on the points on the contour for the computation of the forces. Thus, as a first step, we recast the computation of the forces as a multiscale transformation.

\section{MSAC TRANSFORMATIONS FOR SEGMENTATION}

While active contour methods are the state-of-the-art tool in the image segmentation community, the unique speckled nature of fluorescence microscope images (see, for example, Fig 1 and Fig. 2) makes the direct application of such techniques difficult. Multiscale methods, on the other hand, are well suited to smooth and extract the significant features of such images.

While the nature of the fluorescence microscope images motivates us to explore the use of multiscale features for their segmentation, our success with the active contour algorithms discussed in Section 2.1.1, encourages us to continue to benefit from the advantages they afford. Recently, there has been an effort to formulate multiscale active contours. ${ }^{23}$ Yet, our motivation as discussed in Section 2.2 stems from the need for an elegant framework that has an efficient implementation. Further, the idea of computing forces at the points on the contour using an FFT implementation has been proposed recently in the context of topology preserving flows. ${ }^{29}$ Yet, this design does not get rid of the time consuming extension function itself. Thus, we come up with the novel idea of using multiscale active contour (MSAC) transformations for segmentation that does not parameterize the geometrically embedded contour.

\subsection{Multiscale Active Contour Transformations}

When confronted by speckled images, such as fluorescence microscope ones, one naturally seeks to connect the dots, that is, to estimate the densities of bright pixels on local neighborhoods of various sizes. Mathematically speaking, the computation of such local densities may be represented as a multiscale smoothing transformation of the original image. To be precise, let $f: \mathbb{R}^{N} \rightarrow \mathbb{R}^{M}$ be a fluorescence microscope image, where $N$ is typically 2 or 3 and $M$ is the number of channels, with each channel corresponding to a different fluorescent probe. Although the proposed techniques will naturally extend to the case $M>1$, for the sake of simplicity, we assume $M=1$ for the remainder of this section. Given a windowing function $g$ of an appropriate degree of smoothness, boundedness, and/or decay (for example, a Gaussian), the corresponding multiscale transformation of $f$ is $W f: \mathbb{R}^{N} \times(0, \infty) \rightarrow \mathbb{R}$,

$$
(\mathrm{W} f)(x ; a)=\int_{\mathbb{R}^{N}} f(x) g\left(\frac{x-y}{a}\right) \mathrm{d} y .
$$




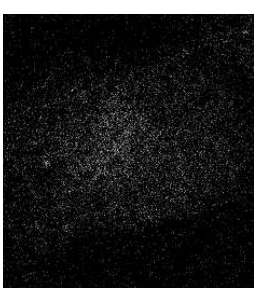

(a)

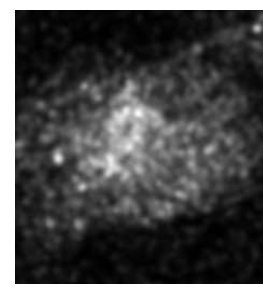

(b)

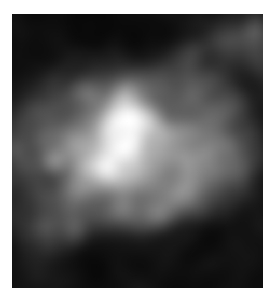

(c)

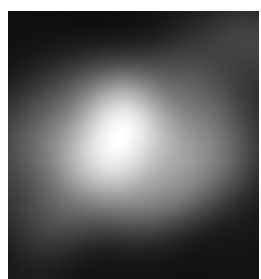

(d)

Figure 6. Multiscale blurs of a fluorescence microscope image. (a) The original image. (b) A slight blur begins to reveal the cell's edge. (c) With more blur, the edge of the cell becomes clearly defined. (d) With too much blur, the edges become rounded.

As depicted in Fig. 6 for several particular scale parameters $a$, such a transformation has a blurring effect, with the blur increasing with $a$. Although blurring is usually regarded as an unwanted artifact in image processing, we see that for fluorescence microscope images, a moderate degree of blur is actually useful: edges and shapes become more apparent as the blur resolves the speckled image. Indeed, more generally, if $f$ were a distribution composed of a weighted sum of Dirac measures, then for each $a>0, \mathrm{Wf}(\cdot ; a)$ would be the sum of translations of a dilated version of $g$. Mathematically speaking, the above transformation (5) is relatively simple, being nothing more than a convolutional operator whose kernel contains a scale parameter. Today, such transformations are commonly called continuous wavelet transforms. Although trivial to prove, it is important to note that transformation (5) preserves the action of the group of rigid transformations, and therefore preserves the underlying geometry of the original image. As microscope images are, in general, taken directly from above, and the vertical axis is the only axis along which we should expect the cells to be aligned, ideally, our algorithms should not be affected by translations, dilations, and rotations.

Specifically, given $x_{0} \in \mathbb{R}^{N}, a_{0}>0$ and an $N \times N$ orthogonal matrix $\Theta$, the corresponding translation, dilation and rotation operators are $\mathrm{T}_{x_{0}}, \mathrm{D}_{a_{0}}, \mathrm{R}_{\theta}: L^{p}\left(\mathbb{R}^{N}\right) \rightarrow L^{p}\left(\mathbb{R}^{N}\right)$,

$$
\left(\mathrm{T}_{x_{0}} f\right)(x)=f\left(x-x_{0}\right), \quad\left(\mathrm{D}_{a_{0}} f\right)(x)=f\left(a_{0}^{-1} x\right), \quad\left(\mathrm{R}_{\Theta} f\right)(x)=f\left(\Theta^{\mathrm{T}} x\right) .
$$

The present day active contour methods are not sensitive to translations, dilations or rotations of the image. Thus, the MSAC transform that we propose, should not introduce these defects and should at the least do no worse than the existing algorithms. Indeed, for any windowing function $g$, the corresponding transformation (5) is easily shown to preserve translations and dilations, according to the following rules:

$$
\left(\mathrm{WT}_{x_{0}} f\right)(x ; a)=(\mathrm{W} f)\left(x-x_{0} ; a\right), \quad\left(\mathrm{WD}_{a_{0}} f\right)(x ; a)=a_{0}^{N}(\mathrm{~W} f)\left(a_{0}^{-1} x, a_{0}^{-1} a\right) .
$$

Transformation such as (5) will also preserve rotations, provided the windowing function $g$ is itself radially symmetric: $g(x)=\widetilde{g}(\|x\|)$ for some $\tilde{g}:[0, \infty) \rightarrow \mathbb{R}$. Then, $\left(\mathrm{WR}_{\Theta} f\right)(x ; a)=(\mathrm{W} f)\left(\Theta^{\mathrm{T}} x ; a\right)$.

Although multiscale transformations have only recently begun to be applied to segmentation problems, ${ }^{23}$ convolution transformations such as (5) (without the scale parameter) have been exploited by the segmentation community for decades. We argue that the scale parameter is essential, as the resolutions of the cameras used in the acquisition process will vary across biological research teams, and, more importantly, as any single fluorescence microscope image will contain multiple regions in which the marked proteins appear with varying density.

\subsection{Segmentation with MSAC Transforms}

The multiscale transform can be combined with the active contour method by suitably coding in the information of the evolving masks. For example, in TPSTACS, the region based force for the fluorescence microscope image is computed as the difference between the sum of the mean densities (or intensities) around a small neighborhood of every point on the current contour and the sum of the precomputed mean models. This may be computed 


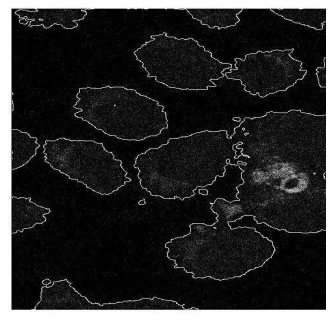

(a)

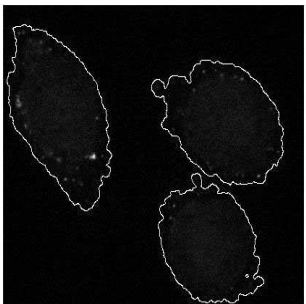

(b)

Figure 7. (a) The total protein image (HeLa-UCE data) after three iterations. (b) The membrane protein image (Golgi data) after three iterations. The merges are due to random initialization and absence of topology preservation.

without looping over the number of points on the contour as follows:

$$
\begin{aligned}
M_{\mathrm{in}} & =\frac{\int_{\mathbb{R}^{N}} f_{\text {in }}(x) g\left(\frac{x-y}{a}\right) \mathrm{d} y}{\int_{\mathbb{R}^{N}} C_{\text {in }}(x) g\left(\frac{x-y}{a}\right) \mathrm{d} y}, \\
M_{\text {out }} & =\frac{\int_{\mathbb{R}^{N}} f_{\text {out }}(x) g\left(\frac{x-y}{a}\right) \mathrm{d} y}{\int_{\mathbb{R}^{N}} C_{\text {out }}(x) g\left(\frac{x-y}{a}\right) \mathrm{d} y} \text { and } \\
F_{r} & =M_{\text {in }}+M_{\text {out }}-M_{\text {model }},
\end{aligned}
$$

where $M_{\text {in }}$ and $M_{\text {out }}$ represent the mean intensities inside and outside the current contour, $M_{\text {model }}=M_{1}+M_{2}$ represents the sum of the model mean intensities $M_{1}$ and $M_{2}, f_{\text {in }}$ represents the portion of the image contained in the current contour, $f_{\text {out }}$ represents the portion that is considered as the background by the current contour, $C_{\text {in }}$ and $C_{\text {out }}$ are the binary masks of the regions inside and outside the contour, respectively, and finally, $F_{r}$ represents the region based force. The behavior of this algorithm at the points on the contour is by design equivalent to the computations performed by TPSTACS. The difference is that instead of using the distance transform and extending the forces thus computed, we actually compute the forces at every pixel in the image obviating the extension function. The values computed cannot be interpreted as their distance from the evolving contour but rather their and their neighboring values' proximity to one mean model or the other. Thus, the computation also enables the contour to evolve much faster as it does not have to progress in small increments. Just using the region based force is insufficient to obtain smooth contours for the cell images. So we designed a new curvature force along the same lines but using the level set function and without computing the second order differentials,

$$
F_{c}=\int_{\mathbb{R}^{N}} \phi(x) g\left(\frac{x-y}{a}\right) \mathrm{d} y-\phi .
$$

The dilation factor $a$ of the smoothing window is selected based on the resolution of the image. With this, we are able to achieve smooth and accurate contours even beginning with a random initialization of the level set function. For example, for both the total protein (HeLa-UCE) and the membrane protein (Golgi) data, if we initialize $\phi$ to be identically zero, we obtain reasonable contours in 3 iterations, each iteration taking about 6.5 seconds on an average (on an Intel Pentium M 1.6 GHz platform) for images of size $1024 \times 1024$. Sample results are shown in Fig. 7. When we have a priori knowledge of the topology, then this method can be easily combined with the topology preservation method used in TPSTACS. This topology preserving module can be expected to increase the run time of the algorithm and this increase is directly proportional to the size and number of contours in the image.

This algorithm is, like its predecessor, extendable to other modalities. A preliminary experiment of the MSAC Transform to segment the brain fMRI images using the appropriate region based force yielded an enormous speedup. The MSAC transform completed segmenting the entire volume of nearly $6002 \mathrm{D}$ images in one hour versus the 36 hours taken by the previous system. As far as accuracy was concerned, it did no worse than the segmentation accuracy of the previous algorithm. 
Note that though there is a provision of using multiple scales to evolve the contour, we have only presented the algorithm and results for a fixed value of the scale. We can combine this algorithm with the multiresolution perspective, that is, smoothing followed by downsampling, and iteratively refine the contour to further increase the algorithm's efficacy and efficiency.

\subsection{The bimagicLab Segmentation Toolbox}

The MSAC transform forms the core of our segmentation algorithm. We propose to select modules such as multiresolution (downsampling and/or use of different dilations of the smoothing filter) and topology preservation based on the details of the data set (such as the imaging modality, dimension of the data set, experimental conditions, etc.) and the user's specific needs. For most of our end users, segmentation is typically the first step towards answering their biological question. They need not concern themselves with all the modules, etc. Thus, we intend to provide a user-friendly graphical user interface for the algorithms we develop. All they would have to do is indicate to the software the experimental conditions such as, for example, the imaging modality, dimensionality, resolution and the nature of the image (such as total protein channel with no parallel channels). The GUI will guide them in the process of selecting the modules most relevant to their data and application. Further, if any parameters need to be tuned explicitly, the GUI will guide the users by providing intuitive descriptions such as the cell size, to facilitate customization. We do recognize that in some tasks, such as high-throughput imaging or high-content screening, 2D images cannot possibly be segmented one at a time. In this aspect, the user investing a few minutes to configure the algorithm through the GUI, will greatly enhance the quality of the segmentation output of the batch processing.

\section{CONCLUSIONS}

We have presented an active contour algorithm that successfully segments fluorescence microscope images. Further, we have demonstrated its flexibility to be adapted to different imaging modalities. It is however not suitable for high-throughput applications due to its large run time. We showed that by incorporating the power of multiresolution, we not only successfully decrease the run time but are also able to detect features that could not be detected earlier and hence segment even those images that do not have any parallel channels for initialization. Furthermore, we proposed a novel multiscale transformation paradigm that can be used to segment images efficiently and without losing the advantage of accuracy and flexibility provided by active contours. This is only the beginning of the work that has raised interesting theoretical and experimental questions and opened new vistas for further exploration.

\section{ACKNOWLEDGEMENTS}

We thank Eric Chu, Philip Cuadra, Siddharth J. Garg, Vivek S. Oak and Sarah J. Reid, for their experimental work in applying the TPSTACS algorithm to other imaging modalities. Our thanks are also due to our collaborators, Dr. R. F. Murphy, Dr. A. D. Linstedt, Dr. B. A. Wandell, Dr. A. F. Laine and their teams for providing us with the images we have used in the preparation of this paper.

\section{REFERENCES}

1. K. Huang and R. Murphy, "From quantitative microscopy to automated image understanding," Journ. Biomed. Optics 9, pp. 893-912, 2004.

2. A. Chebira, Y. Barbotin, C. Jackson, T. Merryman, G. Srinivasa, R. Murphy, and J. Kovačević, "A multiresolution approach to automated classification of protein subcellular location images," BMC Bioinformatics 8(210), 2007.

3. M. Velliste and R. Murphy, "Automated determination of protein subcellular locations from 3D fluorescence microscope images," in Proc. IEEE Int. Symp. Biomed. Imaging, pp. 867-870, (Washington, DC), 2002.

4. L. Coulot, H. Kischner, A. Chebira, J. Moura, J. Kovačević, E. Osuna, and R. Murphy, "Topology preserving STACS segmentation of protein subcellular location images," in Proc. IEEE Int. Symp. Biomed. Imaging, pp. 566-569, (Arlington, VA), Apr. 2006. 
5. M. Puthenveedu and A. Linstedt, "Subcompartmentalizing the Golgi apparatus," Curr. Opin. Cell Biol 17(4), pp. 369-75, 2005.

6. Y. Guo and A. Linstedt, "COPII-Golgi protein interactions regulate COPII coat assembly and Golgi size," Journ. Cell Biol. 174(1), pp. 53-56, 2006.

7. S.-C. Chen, T. Zhao, G. Gorden, and R. Murphy, "A novel graphical model approach to segmenting cell images," in Proc. BMES Annual Fall Meeting, 2006.

8. E. Angelini, A. Laine, S. Takuma, J. Holmes, and S. Homma, "LV volume quantification via spatiotemporal analysis of real-time 3-d echocardiography," IEEE Trans. Med. Imag. 20, pp. 457-469, 2001.

9. B. Wandell, A. Brewer, and R. Dougherty, "Visual field map clusters in human cortex," Philosoph. Trans. of the Royal Soc. B: Biol. Sci. 360(1456), pp. 693-707, 2005.

10. S. Beucher, "The watershed algorithm applied to image trasnformation," Scanning Micrscopy 6, pp. 299314, 1992.

11. C. Wählby, Algorithms for Applied Digital Image Cytometry. PhD thesis, Uppsala University, Uppsala, Sweden, 2003.

12. C. Pluempitiwiriyawej, J. Moura, Y. Wu, and C. Ho, "STACS: A new active contour scheme for cardiac MR image segmentation," IEEE Trans. Med. Imag. 24, pp. 593-603, May 2005.

13. J. A. Sethian, Level Set Methods and Fast Marching Methods, Cambridge University Press, 1999.

14. A. Sarti, C. O. Solorzano, S. Lockett, and R. Malladi, "A geometric model for 3-D confocal image analysis," IEEE Trans. Biomed. Eng. 47(12), pp. 1600-1609, 2000.

15. B. Appleton and H. Talbot, "Globally optimal geodesic active contours," Journ. Math. Imag. Vis. 23(1), pp. 67-86, 2005.

16. G. Srinivasa, A. Chebira, L. Coulot, H. Kirshner, V. Oak, J. Moura, R. Murphy, and J. Kovačević, "A flexible active contour algorithm for the segmentation of fluorescence microscope cell images," BMC Bioinformatics , 2007. To be submitted.

17. X. Han, C. Xu, and J. Prince, "A topology preserving level set method for geometric deformable models," IEEE Trans. Patt. Anal. and Mach. Intelligence 25, pp. 755-768, Jun. 2003.

18. A. Zijdenbos, B. Dawant, R. Margolin, and A. Palmer, "Morphometric analysis of white matter lesions in MR images: Method and validation," IEEE Trans. Med. Imag. 13, pp. 716 - 724, Dec. 1994.

19. M. Vetterli and J. Kovačević, Wavelets and Subband Coding, Signal Processing, Prentice Hall, Englewood Cliffs, NJ, 1995.

20. P. de Rivaz and N. Kingsbury, "Fast segmentation using level set curves of complex wavelet surfaces," in Proc. IEEE Int. Conf. Image Proc., (Vancouver, Canada), Sep. 1998.

21. H. Zhang, Z. Bian, Y. Guo, B. Fei, and M. Ye, "An efficient multiscale approach to level set evolution," in Proc. IEEE Int. Conf. EMBS Society, 1, pp. 694-697, 2003.

22. P.-Y. Tsang, Multi-resolution Image Segmentation Using Active Contours. PhD thesis, University of Waterloo, 2004.

23. X. Bresson, P. Vandergheynst, and J.-P. Thiran, "Multiscale active contours," Int. Journ. Comp. Vis. 70(3), pp. 197-211, 2006.

24. R. Howson, W. Huh, S. Ghaemmaghami, J. Falvo, K. Bower, A. Belle, N. Dephoure, D. Wykoff, J. Weissman, and E. O'Shea, "Construction, verification and experimental use of two epitope-tagged collections of budding yeast strains: Research papers," Comparative and Functional Genomics 6, pp. 2-16, Feb. 2005.

25. J. R. J. Alaniz, V. M. B. nuelos, and O. Y. Suárez, "Data-driven brain MRI segmentation supported on edge confidence and a priori tissue information," IEEE Trans. Med. Imag. 25(1), 2006.

26. J. S. Duncan, X. Papademetris, J. Yang, M. Jackowski, X. Zeng, and L. Staib, "Geometric strategies for neuroanatomic analysis from MRI," NeuroImage 23, pp. S34-S45, 2004.

27. P. C. Teo, G. Sapiro, and B. A. Wandell, "Creating connected representations of cortical gray mater for functional MRI visualization," IEEE Trans. Med. Imag. 16(6), pp. 852-863, 1997.

28. J. J. Bartko, "Measurement and reliability: Statistical thinking considerations," Schizophrenia Bullet. 17(3), pp. 483-489, 1991.

29. G. Sundaramoorthi and A. Yezzi, "Global regularizing flows with topology preservation for active contours and polygons," tech. rep., Georgia Institute of Technology, Aug. 2005. 